co-authors. It was solely R.C. Gallo's decision that the composite be shifted from another paper to mine.

J. SCHÜPBACH Swiss Retrovirus Reference Laboratory, Institut für Immunologie und Virologie, Universität Zürich,

Gloriastrasse 30,

Postfach CH-8028 Zürich, Switzerland

1. Beardsley, T. Nature 320, 563 (1986).

2. Gilden, R.V. et al. Science 232, 307 (1986)

3. Schüpbach, J. et al. Science 224, $503-505$ (1984).

\section{Keeping supercomputers under control}

SIR-The Purdue Water Resources Reseach Center in conjunction with the Office of Health and Environmental Research of the US Department of Energy recently co-sponsored a meeting on the current uses of, and future needs for, supercomputers in hydrology. 'Supercomputers in Hydrology: Future Directions', the first meeting of its kind, aimed to evaluate the need for large-scale vector and parallel processing machines and their peripherals in the hydrologic sciences, especially in subsurface hydraulics. While the charge of the seminar was to specifically deal with hydrologic applications of supercomputers, the conclusions drawn at the meeting apply equally well to many other fields of science and technology.

Almost all technical issues involving the use of supercomputers were presented either by a computer scientist, a programmer or an applied mathematician. Because the architecture of most supercomputers is radically different from scalar processing machines, this is no surprise. The real importance of this observation is the implied increase in the need for multidisciplinary research teams to solve major ecological problems. Unfortunately, in the United States, many of the larger supercomputer funding initiatives do not address this issue at all.

This defect in the supercomputer initiatives leads to two major problems: scientists not doing 'science' and the inefficient use of supercomputers. If funds were available to support programmers with expertise on a machine of a certain architecture, the scientist would only need to communicate effectively with the programmer. The projected changes in architecture affect the programmer and not the scientist; hence the scientist is free to do science.

An alternative to long-run funding of programmer-consultants is for the initiatives to increase their effort in the development of very high level languages. Rice of Purdue's Computer Science Department gave a talk on projected advances in hardware and software over the next 10 years. From 1975 to 1985 computing speed increased by a factor of 25 . Between 1985 and 1995 the speed is projected to increase by a factor of 2,000 . On the other hand, from 1975 to 1985 , computer languages have been improved by a factor of only 1.4. Unless more funding is made available for the development of high-level languages, improvement in ease of coding is not expected to increase nearly as fast as machine computational power. In fact, because of radical changes in architecture, programming may well become more difficult.

Another important point brought out at the seminar is that advances in science should not be overpowered by advances in computational capability. Do we really improve our fundamental understanding of science by looking at larger and larger computational problems? Many would argue that the understanding of complex chemical and physical interactions in the subsurface and the ability to estimate parameters is so poor that the large supercomputer models may in fact be totally incorrect. In this instance, supercomputer modelling efforts may turn out to be a greater obstacle to real progress than no model at all.

So there is clearly a role for supercomputers, but the extent of this role will depend on the availability of high level languages, expert programmer consultants working closely with multidisciplinary teams, exposure to different computational systems, and a judicious choice of problems with the aim of advancing our understanding of science.

JoHn H. Cushman

Water Resources Research Center,

Lilly Hall of Life Sciences,

Purdue University,

West Lafayette,

Indiana 47907, USA

Frank J. Wobber

US Department of Energy,

Office of Energy Research,

OHER (ER-75),

Washington, DC 20545, USA

\section{Are light head loads carried free?}

SIR-Geoffrey Maloiy and his colleagues (Nature 319, 668; 1986) have shown conclusively that experienced African women can carry head loads at remarkably low energy cost. However, I cannot accept their conclusion that loads of less than 20 per cent of body mass are carried free, on the basis of the evidence they present. They performed no measurements in the load range 0 to 20 per cent: their conclusion is based on the backward extrapolation of a straight regression line fitted to the range of about 24 to 70 per cent of body mass. Extrapolation of a regression line always is risky, and in their case particularly so, because other data they present clearly show that the energy cost to army recruits of carrying a back pack is not a linear function of load.

Maloiy and his colleagues suggest that an explanation for the low cost is that the women achieve a gait such that the load makes little movement against gravity during the walking cycle. Though proof of this theory using accelerometers, as R. McNeill Alexander proposes in his News and Views piece on the paper (Nature 319, $623 ; 1986$ ), would be elegant, my observations of African women carrying head loads leads me to believe that they are right. Many South African women can carry small objects like individual oranges or bottles on their heads with grace and ease; some do so by choice even when their hands are free. While it is possible to carry a metastable object like a bottle on one's head even when one's head is moving up and down, the women appear to reduce or eliminate all vertical and transverse head movements when doing so. Given the human anatomy, I think that stabilizing the head necessarily must stabilize the centre of gravity of the whole body too. Since fuelling of cyclic vertical movements of the centre of gravity constitutes one component of the energy cost of walking, elimination of such movements should reduce the energy cost. Thus, the cost of walking with a light load on the head, in people experienced in the skill, might be less than the cost of walking with no load.

We need the missing measurements. Duncan Mitchell

Department of Physiology,

University of the Witwatersrand Medical School,

Johannesburg 2001,

South Africa

SIR-As supporting evidence for Maloiy et al.'s suggestion (Nature 319, 668; 1986) about the steadiness of a head load, I cite the observation that even small children can carry liquid loads on their heads without spilling from the container. A woman carrying a bucket of water home from the well is surely one of the commonest sights; and I would think a good substitute for the suggested experiment of attaching an accelerometer to the head load.

It is interesting that it is possible to carry a much heavier load on the head than the bearer can lift unaided. It is normal to see two people lifting a burden and settling it comfortably on the head of a third, who then walks off with it. One of the most impressive, if macabre, sights I ever saw was when a farmer had died in the field, and another carried the corpse, by then rigid, to the village on his head.

\section{Department of Chemistry and Applied \\ Chemistry,}

University of Natal,

Durban 4001

South Africa 